\title{
Ubiquitous RNA-dependent RNA polymerase and gene silencing
}

\author{
James A Birchler
}

Address: Division of Biological Sciences, University of Missouri, Columbia, MO 65211, USA. Email: BirchlerJ@Missouri.edu

\begin{abstract}
The discovery of a novel RNA-dependent RNA polymerase activity with a eukaryote-wide distribution raises new questions about the roles and mechanisms of gene silencing by small RNAs.
\end{abstract}

The phenomenon of the silencing of transgenes together with homologous genes in the host genome was first discovered some two decades ago in plants [1-3]. This silencing was eventually found to involve either posttranscriptional destruction of the transgene mRNAs $[2,3]$ or prevention of their transcription [1]. The biological rationale for these processes was proposed to be a defense against viruses and transposable elements. Doublestranded RNA (dsRNA) is a target of the silencing mechanism and many viruses produce such RNA as part of their life cycle. Similarly, transposable elements produce aberrant RNAs that enter the silencing pathway and this prevents transposition. This host defense concept has since been supported by a wide variety of evidence. The independent discovery in Caenorhabditis elegans that introduced 'sense' RNAs could cause gene silencing led to the development of the technique of RNA interference (RNAi) [4]. The finding that the active ingredient in triggering RNAi was a dsRNA ignited a revolution in genetics that continues to this day [5].

An interesting aspect of post-transcriptional silencing in plants and worms is that silencing can spread throughout the organism from the site of introduction of the dsRNA. This spread depends on the activity of an RNA-dependent RNA polymerase (RdRP), which amplifies dsRNA to produce more copies, which are then cleaved by the enzyme Dicer to produce small interfering RNAs (siRNAs) [6] that target mRNAs of similar sequence for destruction. The genomes of Drosophila and mammals show no evidence of such an RdRP, and the spread of RNAi from one tissue to another is indeed limited in these animals. Nevertheless, other aspects of post-transcriptional silencing suggested that some type of RdRP activity might be critical in flies and humans as well. For example, aberrant single-stranded RNAs (ssRNAs) are also subject to silencing and so presumably must be converted to a doublestranded form $[7,8]$. Indeed, an RdRP activity was reported in extracts of Drosophila some years ago [9], but the conventional wisdom based on the genomic data held that such an activity did not exist in flies and mammals.

In a recent report, Lipardi and Paterson [10] now define a gene in Drosophila whose product has a newly recognized RNA-dependent RNA polymerase activity, which provides a potential solution to the outstanding issues in posttranscriptional silencing noted above. Moreover, the authors show that this gene product is a multifunctional protein, which in one of its roles is a member of the transcriptional RNA polymerase II elongator complex, and that, unlike the canonical RdRP, it is present in all eukaryotes examined so far. Its ubiquity raises many interesting questions about other possible roles for silencing by small RNAs other than as a defense against viruses and transposons.

\section{Elp1 is a novel RNA-dependent RNA polymerase}

Lipardi and Paterson [10] sought to purify the previously reported RdRP activity [9] from Drosophila embryo extracts. They then tested the candidate proteins identified from a partial purification to determine whether they could inhibit RNAi of green fluorescent protein (GFP) in cultured Drosophila cells engineered for that purpose. They found that knockdown of the Drosophila homolog of the RNA polymerase II core elongator complex subunit Elp1 in the cultured cells inhibited RNAi. Knockdown of other members of the elongator complex had no effect on GFP silencing, and ectopically expressed Elp1 protein was clearly visualized in the cytoplasm. The authors produced recombinant Elp1 protein and showed that it could generate dsRNA using ssRNA as a template in both primerindependent and primer-dependent reactions in vitro. They then coexpressed different combinations of Elp1, Argonaute-2 (which binds siRNAs), Dicer-2 (which cleaves dsRNA to siRNAs) and Dicer-1 (which cleaves microRNA precursors to the mature form) in cultured Drosophila cells, and looked for protein-protein interactions. The strongest interaction was between Elp1 and Dicer-2, which demonstrates a connection to RNAi given the central role of Dicer-2 in producing siRNAs. The levels of certain microRNAs were assayed in Elp1-depleted cells and found not to be affected, indicating a specificity of Elp1 for the RNAi pathway. 
Knockdowns of Elp1 by RNAi [10] produced an increase in the expression of a set of retrotransposons, which were similarly affected by knockdown of Dicer-2. A corresponding reduction in the levels of siRNAs for these transposons was also found. RNAi knockdown of Elp1 specifically reduced the levels of antisense RNAs for the affected transposons, whereas depletion of Dicer-2 did not, thus strengthening the argument that Elp1 provides an RNAdependent RNA polymerase activity that generates the antisense strand. Unlike the gene for canonical RdRP, the elp1 gene is conserved in all eukaryotes examined [10]. Recombinant Elp1 produced from fission yeast, C. elegans and human sequences was shown to have RdRP activity in vitro. Thus, this activity is present in species with and without the canonical RdRP gene.

The elucidation of an RdRP activity that can act without a primer helps to clarify findings that 'aberrant' ssRNAs can be degraded by the silencing process $[7,8,11]$. This posttranscriptional silencing in the absence of an antisense copy or a foldback structure that could produce a dsRNA to serve as a substrate for Dicer had previously posed a conundrum. The discovery of Elp1 RdRP activity provides a possible solution, given that it could potentially convert the aberrant ssRNAs to a double-stranded structure without an antisense primer, but such a scenario needs to be tested experimentally.

\section{Questions for the future}

Small RNAs are also involved in transcriptional silencing $[7,11,12]$, and there is increasing evidence that interaction of RNA polymerase II (PolII) with the small RNA machinery is intimately involved in this silencing. The first indication came from fission yeast (Schizosaccharomyces pombe) in which mutation in the largest and the second largest of the polymerase subunits impaired transcriptional silencing $[13,14]$. In plants, novel RNA polymerases [15] have been found that are dedicated to small-RNA-based transcriptional silencing. The finding of Lipardi and Paterson [10] of a protein with RdRP activity associated with the PolII core elongator complex raises the question of whether this protein is involved in transcriptional silencing. It may indeed play no such role in the polymerase complex, given that it has a cytoplasmic localization when involved in post-transcriptional silencing. However, the evidence of a role for PolII in silencing suggests that investigating the possibility would be an important future direction for work on gene silencing.

Somewhat ironically, the Elp1 protein has a much broader phylogenetic distribution among eukaryotes than the so-called 'canonical' RdRP [10]. So another important question raised by these findings is whether the Elp1 RdRP activity is present in all eukaryotes, including those with the canonical RdRP, and what is the relationship of the two activities in gene-silencing mechanisms? The fact that mutations in the canonical gene produce a recognizable effect on silencing is a classic genetic argument that the two functions will not be completely redundant. Nevertheless, this deserves more investigation.

In addition to the siRNA products of Dicer, Drosophila and mammals produce other short RNAs called piRNAs that associate with the PIWI family of Argonaute proteins [16-18]. piRNAs are found in abundance in germline cells but have also been reported in somatic tissues. One proposed role of these small RNAs is as a defense against transposable elements. The terminal structure of piRNAs is not like that produced by Dicer [19] and so they are likely to be produced by a Dicer-independent mechanism. Different members of the PIWI protein family show a preference for binding to either the sense or antisense versions of retrotransposon piRNAs [17,18]. The complementary sequence arrangements of piRNAs had suggested that the sense and antisense versions serve as guides for different Argonaute proteins to cleave them to their characteristic 25-bp length. The presumed absence of an RdRP activity in flies and mammals figured in the development of this idea because there was no known mechanism by which dsRNA intermediates could be generated. With the revelation that such an activity exists, an interesting direction of investigation would be to test whether production of piRNAs does in fact involve a dsRNA intermediate. Indeed, given that piRNAs are implicated in transcriptional silencing $[20,21]$, interconnections among PolII, Elp1 and the PIWI family of Argonaute proteins in silencing processes would be worth exploring.

Finally, the finding of a protein with RdRP activity that associates with PolII and is found in all eukaryotes might suggest an expansion of the raison d'être for small RNAmediated silencing mechanisms. It might well be that small RNAs have functions in RNA proofreading and quality control that act to silence the transcription of DNA sequences encoding unwanted or aberrant RNAs. This mechanism would silence transposable elements as just one aspect of its broader role in gene expression. Nevertheless, the antiviral functions of small RNAs act post-transcriptionally and the relationship of these to transcriptional silencing is still in need of clarification. These potential interconnections raise new questions for the ever-enlarging field of small RNAs.

\section{Acknowledgements}

Research support from NSF MCB 0923607 is gratefully acknowledged.

\section{References}

1. Matzke MA, Primig M, Trnovsky J, Matzke AJM: Reversible methylation and inactivation of marker genes in sequentially transformed tobacco plants. EMBO J 1989, 8:643-649.

2. Napoli C, Lemieux C, Jorgenson R: Introduction of a chimeric chalcone synthase gene in Petunia results in 
reversible co-suppression of homologous genes in trans. Plant Cell 1990, 2:279-289.

3. Van der Krol AR, Mur LA, Beld M, Mol JNM, Stuitje AR: Flavonoid genes in Petunia: addition of a limited number of gene copies may lead to a suppression of gene expression. Plant Cell 1990, 2:291-299.

4. Guo S, Kemphues K: par-1, a gene required for establishing polarity in C. elegans embryos, encodes a putative Ser/Thr kinase that is asymmetrically distributed. Cell 1995, 81:611620.

5. Fire A, Xu S, Montgomery MK, Kostas SA, Driver SE, Mello $\mathrm{CC}$ : Potent and specific genetic interference by doublestranded RNA in Caenorhabditis elegans. Nature 1998, 391: 806-811.

6. Hamilton AJ, Baulcombe DC: A species of small antisense RNA in posttranscriptional gene silencing in plants. Science 1999, 286:950-952.

7. Pal-Bhadra M, Bhadra U, Birchler JA: RNAi related mechanisms affect both transcriptional and post-transcriptional transgene silencing in Drosophila. Mol Cell 2002, 9:315327.

8. Siomi MC, Tsukumo $H$, Ishizuda A, Nagami T, Siomi $H$ : A potential link between transgene silencing and poly (A) tails. RNA 2005, 11:1004-1011.

9. Lipardi $C$, Wei $Q$, Paterson $B M$ : RNAi as random degradative PCR: siRNA primers convert mRNA into dsRNAs that are degraded to generate siRNAs. Cell 2001, 107:297-307.

10. Lipardi C, Paterson BM: Identification of an RNA-dependent RNA polymerase in Drosophila involved in RNAi and transposon suppression. Proc Natl Acad Sci USA 2009, 106: 15645-15650.

11. Mette MF, van der Winden J, Matzke MA, Matzke AJM: Production of aberrant promoter transcripts contributes to methylation and silencing of unlinked homologous promoters in trans. EMBO J 1999, 18:241-248.

12. Volpe TA, Kidner C, Hall IM, Teng G, Grewal SI, Martienssen RA: Regulation of heterochromatic silencing and histone H3 lysine-9 methylation by RNAi. Science 2002, 297:18331837.

13. Schramke V, Sheedy DM, Denli AM, Bonila C, Ekwall K, Hannon GJ, Allshire RC: RNA-interference-directed chroma- tin modification coupled to RNA polymerase II transcription. Nature 2005, 435:1275-1279.

14. Kato $\mathrm{H}$, Goto DB, Martienssen RA, Urano T, Furkawa $\mathrm{K}$, Murakami Y: RNA polymerase II is required for RNAidependent heterochromatin assembly. Science 2005, 309: 467-469.

15. Onodera T, Haag JR, Ream T, Nunes PC, Pontes O, Pikaard CS: Plant nuclear RNA polymerase IV mediates siRNA and DNA methylation-dependent heterochromatin formation. Cell 2005, 120:613-622.

16. Aravin AA, Hannon GJ, Brennecke J: The Piwi-piRNA pathway provides an adaptive defense in the transposon arms race. Science 2007, 318:761-764.

17. Brennecke J, Aravin AA, Stark A, Dus $M$, Kellis $M$, Sachidanandan R, Hannon GJ: Discrete small RNAgenerating loci as master regulators of transposon activity in Drosophila. Cell 2007, 128:1089-1103.

18. Gunawardane LS, Saito K, Nishida KM, Miyoshi K, Kawamura $\mathrm{Y}$, Nagami T, Siomi H, Siomi MC: A slicer-mediated mechanism for repeat-associated siRNA 5 , end formation in Drosophila. Science 2007, 315:1587-1590.

19. Vagin VV, Sigova A, Li C, Seitz H, Gvozdev V, Zamore P: A distinct small RNA pathway silences selfish genetic elements in the germline. Science 2006, 313:320-324.

20. Brower-Toland B, Findley SD, Jiang L, Liu L, Yin H, Dus M, Zhou P, Elgin SCR, Lin H: Drosophila PIWI associates with chromatin and interacts directly with HP1a. Genes Dev 2007, 21:2300-2311.

21. Kuramochi-Miyagawa $\mathrm{S}$, Watanabe $\mathrm{T}$, Gotoh $\mathrm{K}$, Totoki $\mathrm{Y}$, Toyoda A, Ikawa M, Asada N, Kojima K, Yamaguchi Y, ljiri TW, Hata K, Li e, Matsuda Y, Kimura T, Okabe M, Sakaki Y, Sasak $\mathrm{H}$, Nakano T: DNA methylation of retrotransposon genes is regulated by Piwi family members MILI and MIWI2 in murine fetal testes. Genes Dev 2008, 22:908-917.

Published: 26 November 2009

doi:10.1186/gb-2009-10-11-243

(C) 2009 BioMed Central Ltd 